REPLY

\title{
Alternative approaches to generating cardiomyocytes are under development
}

\author{
Ian Y. Chen, Elena Matsa and Joseph C. Wu
}

We would like to thank Masuda et al. for their Correspondence (Expandable progenitors from induced pluripotent stem cells. Nat. Rev. Cardiol. doi:10.1038/nrcardio.2016.129) ${ }^{1}$ on our Review (Induced pluripotent stem cells: at the heart of cardiovascular precision medicine. Nat. Rev. Cardiol. 13, 333-349; 2016) ${ }^{2}$. We agree with Masuda and colleagues that the current approaches to generating induced pluripotent stem cells (iPSCs) and iPSCderived cardiomyocytes (iPSC-CMs) can be both time-consuming and costly. Furthermore, the resultant cell products can exhibit batch-to-batch variations, making it difficult to interpret subtle phenotypic changes in iPSCbased applications, including drug screening, disease modelling, and regenerative therapy. The recent development of techniques to isolate, maintain, and substantially expand mouse and human induced cardiovascular progenitor cells (iCPCs) in culture has provided alternative methods to produce cardiomyocytes that are potentially less heterogeneous than iPSC-CMs and in manners that are potentially less time-consuming and costly. We applaud Masuda et al. for discussing these promising techniques in their Correspondence. For those interested in reading further on this topic, we gladly refer the readers to our recent editorial in Circulation Research, which additionally discusses the necessary steps as well as hurdles for the clinical translation of expandable iCPCs ${ }^{3}$.

Ian Y. Chen, Elena Matsa and Joseph C. Wu are at the Stanford Cardiovascular Institute and the Department of Medicine, Division of Cardiovascular Medicine, Stanford University School of Medicine.

Elena Matsa and Joseph C. Wu are at the Department of Radiology, Stanford University School of Medicine, 265 Campus Drive, Stanford, California 94305, USA.

Correspondence to J.C.W. joewu@stanford.edu

doi: 10.1038/nrcardio.2016.130

Published online 19 Aug 2016

1. Masuda, S. et al. Expandable progenitors from induced pluripotent stem cells. Nat. Rev. Cardiol. http://dx.doi.org/10.1038/nrcardio.2016.129 (2016).

2. Chen, I. Y., Matsa, E. \& Wu, J. C. Induced pluripotent stem cells: at the heart of cardiovascular precision medicine. Nat. Rev. Cardiol. 13, 333-349 (2016).

3. Chen, I. Y. \& Wu, J. C. Finding expandable induced cardiovascular progenitor cells. Circ. Res. 119, 16-20 (2016).

Competing interests statement

The authors declare no competing interests. 